\title{
Frequent aberrant methylation of the imprinted IGF2/H19 locus and LINE1 hypomethylation in ovarian carcinoma
}

\author{
REINHARD H. DAMMANN ${ }^{1}$, SEBASTIAN KIRSCH ${ }^{2,3}$, UNDRAGA SCHAGDARSURENGIN ${ }^{1}$, \\ TEMUUJIN DANSRANJAVIN ${ }^{1}$, ELISE GRADHAND ${ }^{2}$, WOLFGANG D. SCHMITT ${ }^{2}$ and STEFFEN HAUPTMANN $^{2}$ \\ ${ }^{1}$ Institute for Genetics, Justus-Liebig University Giessen, D-35392 Giessen; ${ }^{2}$ Institute for Pathology, \\ Martin-Luther University Halle-Wittenberg, D-06097 Halle, Germany
}

Received September 7, 2009; Accepted October 20, 2009

DOI: 10.3892/ijo_00000488

\begin{abstract}
Epigenetic alteration of tumor-related genes through changes of DNA methylation is a hallmark for carcinogenesis and aberrant DNA methylation modulates the activity of tumor suppressor genes, imprinted genes and repetitive elements. In ovarian carcinoma, frequent loss of imprinting or aberrant methylation of repetitive elements were reported, however, combined analysis were not performed. We analyzed the aberrant methylation of a differentially methylated region (DMR0) and a CTCF binding site of the IGF2-H19 locus and methylation of LINE1 and Satellite 2 in 22 primary ovarian carcinomas (OC) and controls by a quantitative bisulfite restriction analysis (QUBRA). In $91 \%$ of OC, a significant hypomethylation of DMR0 was found compared to controls $(\mathrm{p}<0.05)$. In $77 \%$ of OC, a hypermethylation of a CTCF binding site was found $(p<0.05)$. A combined hypomethylation of DMR0 and hypermethylation of the CTCF binding was observed in $73 \%$ of OC. Hypomethylation of LINE1 and Satellite 2 was detected
\end{abstract}

Correspondence to: Dr Reinhard Dammann, Institute for Genetics, Justus Liebig University Giessen, Heinrich-Buff-Ring 58-62, D-35392 Giessen, Germany

E-mail: reinhard.dammann@gen.bio.uni-giessen.de

Dr Steffen Hauptmann, Institute for Pathology, Martin Luther University Halle-Wittenberg, Magdeburger Str. 14, D-06097 Halle (Saale), Germany

E-mail: steffen.hauptmann@medizin.uni-halle.de

Present address: ${ }^{3}$ Center for Cardiovascular Research, Institute of Pharmacology, Charité, Berlin, Germany

Abbreviations: IGF2, insulin-like growth factor 2; DMR, differentially methylated region; ICR, imprinting control region; CTCF, CCCTC-binding factor; BS, binding site; LOI, lost of imprinting; LINE1, long interspersed nuclear element 1; QUBRA, quantitative bisulfite restriction analysis; 5-aza-dCR, 5-aza-2'-deoxycytidin; OC, ovarian carcinoma

Key words: ovarian carcinoma, DNA methylation, insulin-like growth factor 2, CCCTC-binding factor, long interspersed nuclear element 1 in 100 and $23 \%$ of OC, respectively. In summary, we found frequent combined aberrant methylation of the IGF2-H19 locus and LINE1 in the vast majority of OC, suggesting that these changes are important events in tumorigenesis.

\section{Introduction}

Aberrant DNA methylation is almost always detectable in human cancers and may contribute to dysregulated gene expression and chromosomal instability (1). In particular, loss of imprinting of human insulin-like growth factor 2 gene (IGF2) is a common genetic alteration in human malignancies and aberrant methylation of $I G F 2 / H 19$ locus has been detected in Wilms' tumors $(2,3)$, ovarian cancers (OC) (4-6), colorectal carcinomas (7-11), prostate cancers (12), osteosarcomas (13), lung adenocarcinomas (14), and head and neck carcinomas $(15,16)$. Loss of imprinting results in an overexpression of the human IGF2 gene, which encodes a potent mitogenic growth factor. This gene is located together with $H 19$ within an imprinting domain of chromosome 11p15.5 (17,18). Physiologically, only the paternally inherited $I G F 2$ allele is transcriptionally active, while $H 19$ is oppositely imprinted and active only on the maternal allele $(17,18)$. In the IGF2 gene a differentially methylated region (DMR0) was identified in exon 3 and between IGF2 and H19 the imprinting control region (ICR) is located, which is recognized by the insulator CTCF $(19,20)$. Aberrant methylation of the IGF2-DMR and the CTCF binding site within the ICR were reported in cancers (2-16).

DNA methylation is an important silencing mechanism for mobile genetic elements, particularly for those elements containing considerable levels of total $\mathrm{CpG}$ dinucleotides such as long interspersed element-1 (LINE1) and pericentromeric satellite region Satellite 2 (Sat2) (21-24). The activation of transposable elements by hypomethylation may result in critical genomic instability by induction of chromosomal recombination and disturbance of transcriptional control mechanism (24-26). Several reports have already analyzed the aberrant methylation of LINE1 and/or Sat2 in human cancers $(21,24,25,27-29)$.

In the present study, we determined the association between aberrant methylation of the IGF2-H19 locus and repetitive elements by application of a new established quantitative bisulfite restriction analysis (QUBRA) PCR protocol to 
evaluate quantitatively methylation status of the $I G F 2$ differentially methylated region (IGF2-DMRO), the CTCF binding site 6 in the ICR of the IGF2-H19 locus (CTCFBS6), as well as Satellite 2 (Sat2) and LINE1 elements in ovarian carcinoma.

\section{Materials and methods}

Tumor probes, cell lines and DNA preparation. Tumor samples and the clinicopathological data were obtained from 22 patients with ovarian carcinoma (Table I). Blood controls were obtained from healthy individuals with no history of cancer. All patients gave a written consent and the study was approved by the local ethics committee of the Medical Faculty. Tumor histology and grading were classified according to the WHO guidelines. Five human ovarian cancer cell lines (SKOV3, OAW42, OVCAR3, CAOV3 and ES2) and U2OS were cultured in RPMI-1640 or DMEM, supplemented with $10 \%$ FCS. Normal human mammary epithelial cells MCF10 were grown in MEGM (Cambrex, Brussels, Belgium) and treated with 5 and $10 \mu \mathrm{M}$ 5-aza-dCR (Sigma, Taufkirchen, Germany) for 4 days. Genomic DNA was extracted from cultured cells and tissues by protein $\mathrm{K}$ digestion and a phenol/ chloroform procedure.

Quantitative bisulfite restriction analysis (QUBRA). To set up a quantitative bisulfite restriction analysis (QUBRA), unmethylated and completely methylated deaminated DNA sequences were cloned and used as standard to generate calibration curves. Therefore, PCR fragments of IGF2-DMR0, CTCF-BS6, Sat2 and LINE1 were produced with unmodified genomic DNA isolated from human fibroblast and primers listed in Table II. PCR was performed with $150 \mathrm{ng}$ of genomic DNA in $25 \mu 1$ reaction, containing $0.2 \mathrm{mM}$ dNTPs, $1.5 \mathrm{mM}$ $\mathrm{MgCl}_{2}, 10$ pmol of each primer and 1.5 units of High Fidelity Taq Polymerase (Roche, Mannheim, Germany). PCR products were cloned in pGEM-T easy (Promega, Mannheim, Germany) and sequenced (Seqlab, Göttingen, Germany). Two micrograms of plasmid DNA was in vitro methylated with 10 units of SSSI methylase (NEB, Frankfurt, Germany) and 1X SAM at $37^{\circ} \mathrm{C}$ for $16 \mathrm{~h}$ or mock-methylated. Mock-methylated and methylated plasmid were purified and treated with a standard bisulfite deamination protocol. Briefly, DNA was denatured with $0.3 \mathrm{M} \mathrm{NaOH}$ at $37^{\circ} \mathrm{C}$ for $15 \mathrm{~min}$. Sodium bisulfite (3.12 M) (Sigma, Taufkirchen, Germany) and $5 \mathrm{mM}$ hydroquinone (Sigma) was added and incubated at $56^{\circ} \mathrm{C}$ overnight. DNA was purified with the Wizard DNA Clean Up System (Promega) according to the manufacturer protocol and eluted in $50 \mu \mathrm{l} \mathrm{H}_{2} \mathrm{O}$. Finally, DNA was treated for $15 \mathrm{~min}$ with $0.3 \mathrm{M}$ $\mathrm{NaOH}$ at $37^{\circ} \mathrm{C}$, precipitated and resuspended in $50 \mu 1 \mathrm{TE}$ buffer. Subsequently, $200 \mathrm{ng}$ of deaminated DNA was amplified with the bisulfite specific primers listed in Table II and PCR conditions described above. PCR fragments were cloned in pGEM-T easy. Completely converted methylated $(\mathrm{CpG})$ and unmethylated $(\mathrm{TpG}) \mathrm{DNA}$ fragments were identified by sequencing and utilized to generate standard curves for QUBRA. Therefore, different ratio of unmethylated and methylated plasmid were produced $(100 \%=1 \mathrm{pg}$ plasmid $)$ and amplified with a ${ }^{32} \mathrm{P}$ end labeled primer. Primer $(20 \mathrm{pmol})$ were labeled with 10 units of T4 polynucleotide kinase (NEB,
Frankfurt, Germany) and 25 pmol ${ }^{32} \mathrm{P}-\gamma$-ATP. DNA was amplified in $25 \mu \mathrm{l}$ reaction buffer containing $0.2 \mathrm{mM}$ dNTPs, $1.5 \mathrm{mM} \mathrm{MgCl}_{2}, 10 \mathrm{pmol}$ of each primer, $0.2 \mathrm{pmol}$ labeled primer and 2.5 units of Taq polymerase (Invitek, Berlin, Germany) at $95^{\circ} \mathrm{C}$ for $30 \mathrm{sec}$, Ta for $30 \mathrm{sec}$, and $72^{\circ} \mathrm{C}$ for $30 \mathrm{sec}$ for 20 cycles or 40 cycles if no semi-nested PCR was performed. For IGF2-DMR0 and CTCF-BS6 a semi-nested PCR was performed using an internal primer with similar conditions as described for the preceding PCR amplification, but for 30 cycles (Table II). For the restriction enzyme analysis, 20 ng of PCR product was digested with 10 units of TaqI or HpyCH4IV (New England Biolabs; Beverly, MA), according to conditions specified by the manufacturer of the enzyme and separated on $10 \%$ polyacrylamid gels with Tris-borate EDTA at $150 \mathrm{~V}$ for $45 \mathrm{~min}$. Gels were dried on filter papers and exposed to a phosphor screen and analyzed with Image Quant 5.0 on a Storm 860 (Molecular Dynamics, Sunnyvale, USA). All experiments were done at least three times. Subsequently, standard curves were determined and a polynomial regression was performed. This function was used to calculate the exact amount of methylated DNA in the tissue samples. Therefore, $2 \mu \mathrm{g}$ of genomic DNA isolated from tissue samples and cell lines was bisulfite modified and QUBRA with $200 \mathrm{ng}$ of bisulfite treated DNA was performed as described above. All experiments were done in triplicates and the amount of methylated DNA was determined utilizing polynomial regression.

Statistical analysis. Statistical analyses were carried out using SPSS14. $\mathrm{P}<0.05$ were considered to be significant.

\section{Results}

Quantitative methylation analysis of IGF2-DMR, CTCF-BS6, Satellite 2 (Sat2) and LINE1. For several technical reasons conventional methylation specific PCR and combined bisulfite restriction analysis are not useful to measure methylation changes that occur in the process of loss of imprinting and during hypomethylation. To overcome this technical limitation we established a quantitative bisulfite restriction analysis (QUBRA) of the IGF2-DMR, CTCF-BS6, Sat2 and LINE1 by modifying combined bisulfite restriction analysis (Fig. 1). To adjust for amplification bias during PCR, we utilized distinct amounts of cloned methylated and unmethylated DNA templates and generated calibration curves for QUBRA (Fig. 2). Interestingly for all four analyzed regions, preferential amplification of methylated DNA was observed (Fig. 2). Subsequently, a polynomial regression was performed and the coefficient of determination was obtained (Fig. 2). This polynomial function was utilized to adjust for amplificational bias in our experiments.

Aberrant methylation of the IGF2-H19 locus in primary ovarian carcinoma. Subsequently, we analyzed the methylation status of IGF2-DMR0 and of a CTCF binding site (CTCF-BS6) at the IGF2-H19 locus in 22 primary ovarian carcinomas (OC), five OC cell lines and controls by QUBRA (Fig. 3). In all five controls, an identical methylation pattern was observed (Table III). In 20 out of $22(91 \%)$ primary OC a significant hypomethylation of IGF2-DMR0 was found 
Table I. Demographic data of the primary ovarial carcinoma.

\begin{tabular}{lccccc}
\hline Case & Stage (FIGO) & Grade & Age & Disease-free survival (month) & Overall survival (month) \\
\hline OC2 & Ic & G1 & 67 & 22.0 & 120.2 \\
OC3 & Ic & G2 & 65 & 49.8 & 65.8 \\
OC4 & IIIc & G2 & 67 & 12.0 & 46.9 \\
OC6 & Ic & G1 & 64 & 31.8 & 64.4 \\
OC13 & IIIc & G2 & 55 & 16.9 & 16.9 \\
OC14 & IIIb & G2 & 50 & 11.5 & 11.5 \\
OC15 & IIIb & G3 & 76 & - & 0.1 \\
OC22 & Ia & G1 & 81 & - & 55.1 \\
OC30 & IIIc & G3 & 49 & 5.5 & 22.8 \\
OC32 & IIIc & G3 & 61 & 13.6 & 17.4 \\
OC37 & Ic & G2 & 59 & 43.0 & 70.5 \\
OC39 & IIIc & G2 & 62 & 4.8 & 20.6 \\
OC41 & IIIb & G3 & 57 & 42.6 & 68.2 \\
OC43 & IIIc & G3 & 73 & 7.9 & 62.9 \\
OC45 & Ia & G2 & 64 & 28.2 & 55.1 \\
OC46 & Ib & G3 & 61 & 25.4 & 69.1 \\
OC49 & IIIc & G3 & 66 & - & 0.0 \\
OC50 & IIIc & G2 & 59 & 7.5 & 19.4 \\
OC51 & IIIc & G3 & 72 & 14.1 & 36.4 \\
OC55 & IIb & G3 & 58 & 64.6 & 64.6 \\
OC56 & IIIc & G3 & 68 & 4.1 & 4.1 \\
OC57 & IIIc & G3 & 72 & 34.6 & 43.2 \\
\hline
\end{tabular}

Table II. Primer sequence, annealing temperature (Ta) and product length.

\begin{tabular}{lll}
\hline Primer & \multicolumn{1}{c}{ Sequence (5'-3') } & Ta product \\
\hline LINE12UN & GGGGGGAGGAGCCAAGATGG & $60^{\circ} \mathrm{C}$ \\
LINE12LN & CGAGCTTCCCGGCTGCTTTG & $493 \mathrm{bp}$ \\
CBS6UN & TGAAGGTTGGGGAGATGGGAGGA & $61^{\circ} \mathrm{C}$ \\
CBS6LN & CGTGACTTGAGTCCCAGGCCATG & $461 \mathrm{bp}$ \\
IGF2UN & CACCCTGGGGCCAAGGCAGT & $61^{\circ} \mathrm{C}$ \\
IGF2LN & CTTGAGGGGTCATGGCACGGAAT & $279 \mathrm{bp}$ \\
SAT2UN2 & CGAACGGACCCGAATGGAATC & $59^{\circ} \mathrm{C}$ \\
SAT2LN2 & TTTGATGTTGATTCCATTCGATTCCA & $251 \mathrm{bp}$ \\
SAT2UBS2 & TGGAATGGAATGGAATAATTTATTGGA & $54^{\circ} \mathrm{C}$ \\
SAT2LBS2 & TCCATTAAATAATAACTCCTTTCATTTCCA & $176 \mathrm{bp}$ \\
LINE1U1 $^{\mathrm{a}}$ & GGGGGGAGGAGTTAAGATGGT & $54^{\circ} \mathrm{C}$ \\
LINE1L2 $^{\mathrm{a}}$ & ACTACTTTATTTACCTAAACAAACCTAAACAA & $482 \mathrm{bp}$ \\
UCTCFA $^{\mathrm{a}}$ & GTGTTTTAGTTTTATGGATGATGGGGAT & $54^{\circ} \mathrm{C}$ \\
LCTCFA $^{\mathrm{a}}$ & ATCCCAAACCATAACACTAAAACCCTC & $408 \mathrm{bp}$ \\
UCTCFB $^{\mathrm{a}}$ & GGGGGTTTTGTATAGTATATGGGTATTTT & $327 \mathrm{bp}$ \\
UIGF2A $^{\mathrm{a}}$ & AAGGTAGTTTTTTTGGGATGTTTATTT & $50^{\circ} \mathrm{C}$ \\
LIGF2B $^{\mathrm{a}}$ & CTCCAAACACCCCCACCTTAA & $227 \mathrm{bp}$ \\
UIGF2B $^{\mathrm{a}}$ & TTTTTTGTTGTATTTTGGATTTAGATTTTTT & $185 \mathrm{bp}$ \\
\hline
\end{tabular}

aPrimer for bisulfite treated DNA. 
A
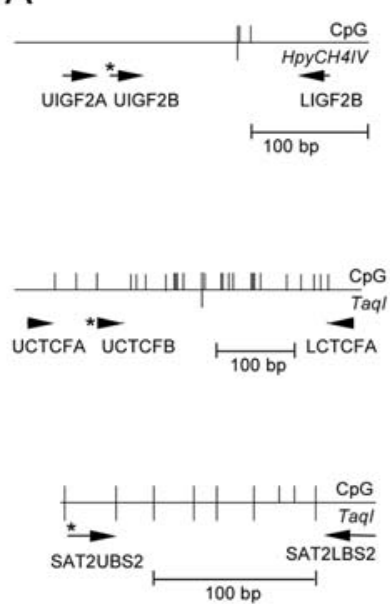

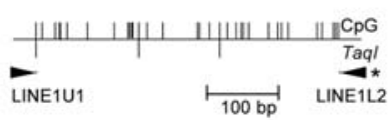

B
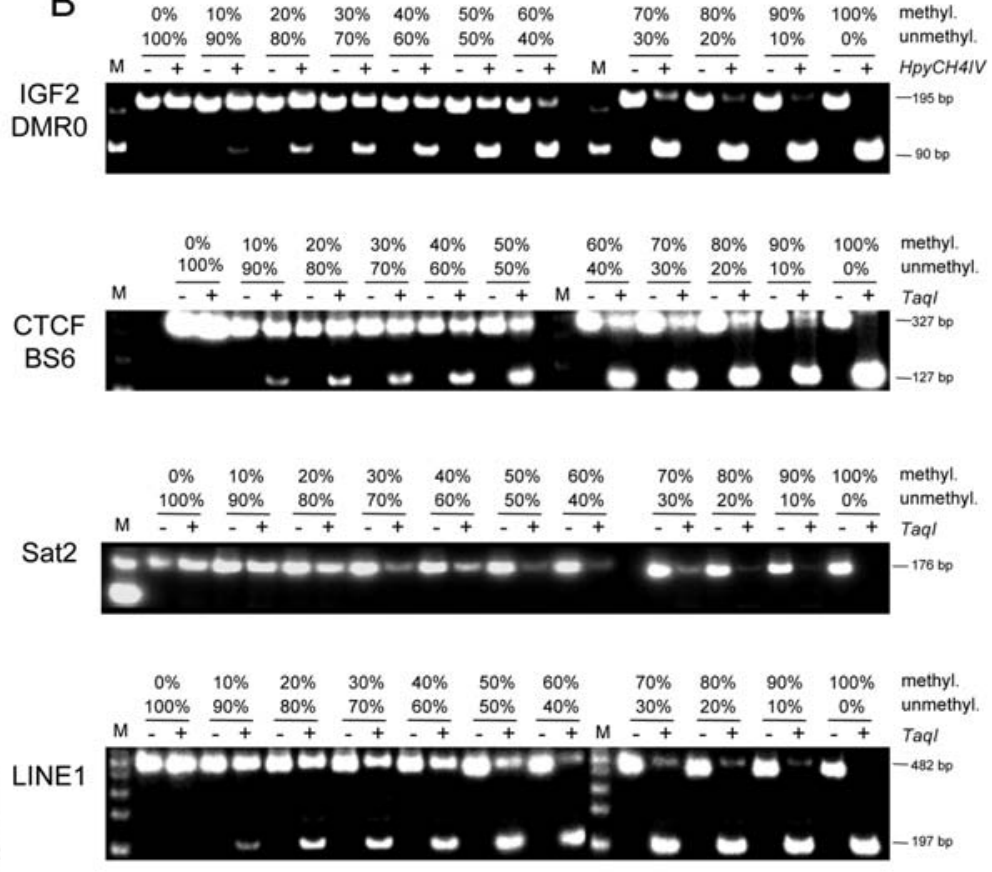

Figure 1. Methylation analysis of IGF2-DMR0, CTCF-BS6, Sat2 and LINE1 by a quantitative bisulfite restiction (QUBRA). (A) Maps of IGF2-DMR, CTCFBS6, Satellite2 (Sat2) and LINE1. Positions of CpGs and of restriction enzyme recognition sites (HpyCh4IV and TaqI) are shown. Arrows indicate utilized primers and labelled primers are marked by asterisks. (B) QUBRA. The indicated amounts of methylated (methyl.) and unmethylated (unmethyl.) DNA standards were mixed and amplified with a labeled primer by PCR. Products were digested (+) or mock-digested (-) with the indicated enzymes and analyzed by PAGE and gels were exposed to phosphors screens. A labeled 100 bp ladder (M) was utilized as a length marker.
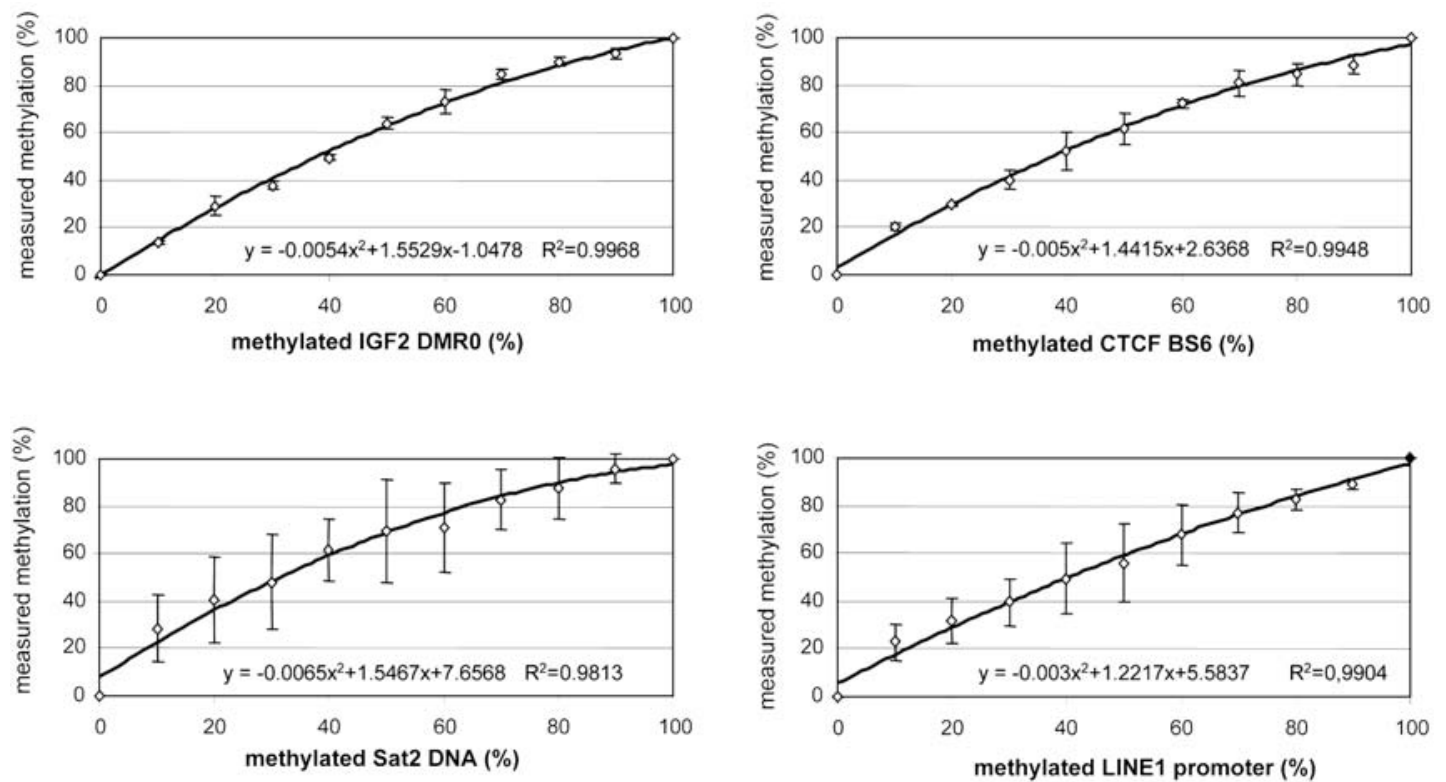

Figure 2. Calibration curves of QUBRA. Utilized and measured percentage of methylated IGF2-DMR0, CTCF-BS6, Sat2 and LINE1 was determined from three independent experiments and plotted on a graph. Standard deviations are indicated. A polynomial regression was performed and for each regression the coefficient of determination was calculated.

compared to controls (p<0.05; Fig. 3A and B; Table III). Moreover, hypermethylation of CTCF-BS6 was observed in 17 out of $22(77 \%)$ OC compared to the controls $(\mathrm{p}<0.05$; Fig. 3C and D; and Table III). A combined hypomethylation of IGF2-DMR0 and hypermethylation of CTCF-BS6 was detected in $73 \%$. In the $\mathrm{OC}$ cell lines the methylation pattern at the IGF2-H19 locus was different compared to primary OC and controls. Three cell lines (SKOV3, OVCAR3 and ES2) showed hypomethylation of both regions and OAW42 and CAOV3 exhibited a hypermethylation of IGF2-DMR0 
A
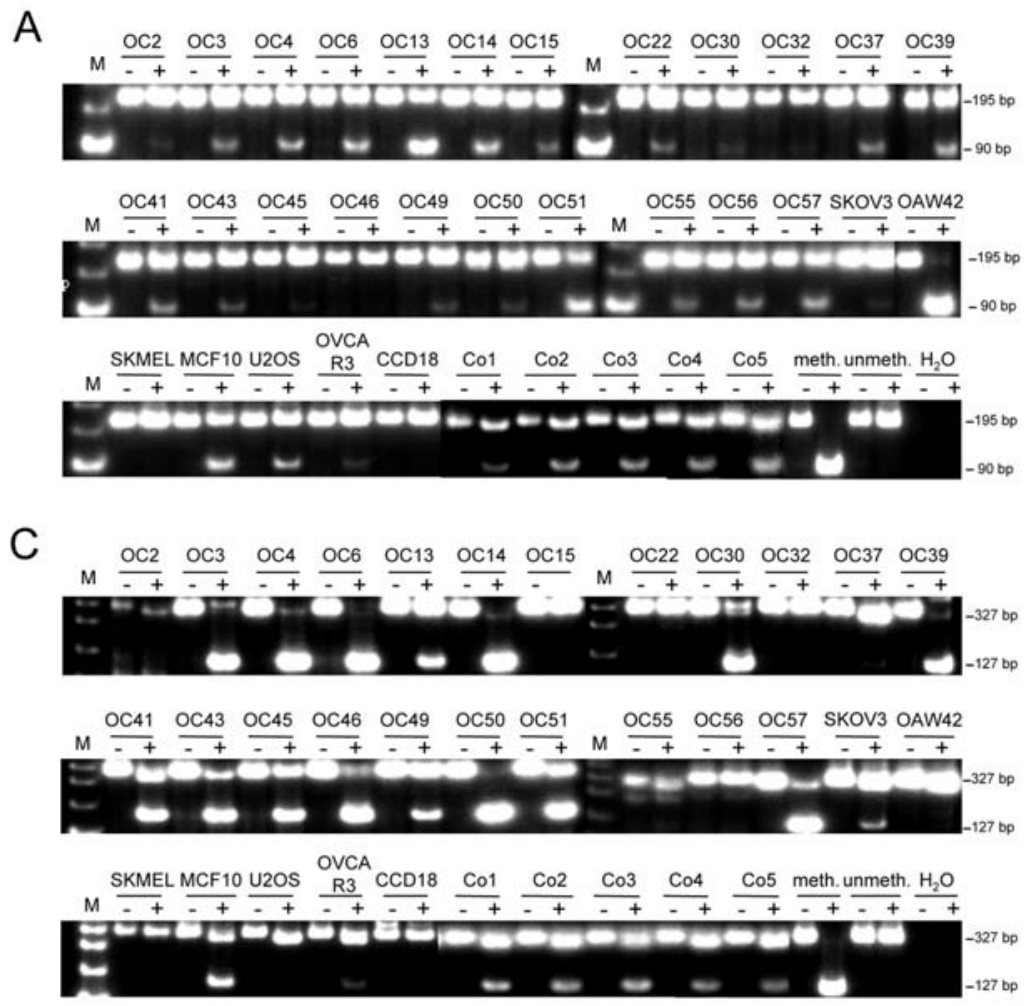
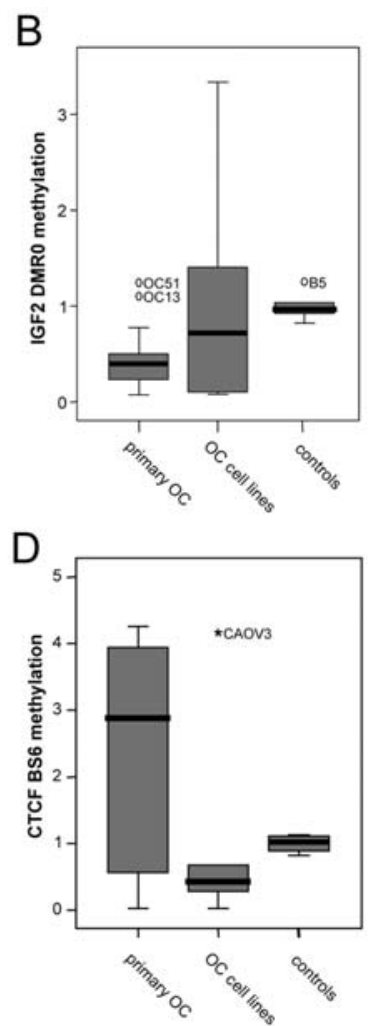

Figure 3. Methylation analysis of IGF2-DMR0 and CTCF-BS in ovarian carcinoma. (A) Methylation of the IGF2-DMR0 in primary ovarian carcinoma (OC) samples, cancer cell lines (SKOV3, OAW42, SKMEL, U2OS and OVCAR3), normal cell lines (MCF10 and CCD18) and controls (Co) was analyzed by QUBRA with restrictions enzyme $(+)$ or mock digest (-). Methylated (meth.) and unmethylated (unmeth.) standards were separated together with a labelled 100 bp ladder (M). (B) Whisker-box-plot of the IGF2-DMR0 methylation status of primary ovarian carcinomas (OC), OC cell lines (SKOV3, OAW42, OVCAR3, CAOV3 and ES2) and five controls (Co). All methylation levels are relative to controls (=1). (C) Methylation analysis of the CTCF-BS6 in primary ovarian carcinoma (OC) samples, cancer cell lines and controls. For details see Fig. 3A. (D) Whisker-box-plot of the CTCF-BS6 methylation in primary ovarian carcinomas (OC), OC cell lines and controls. For details see Fig. 3B.

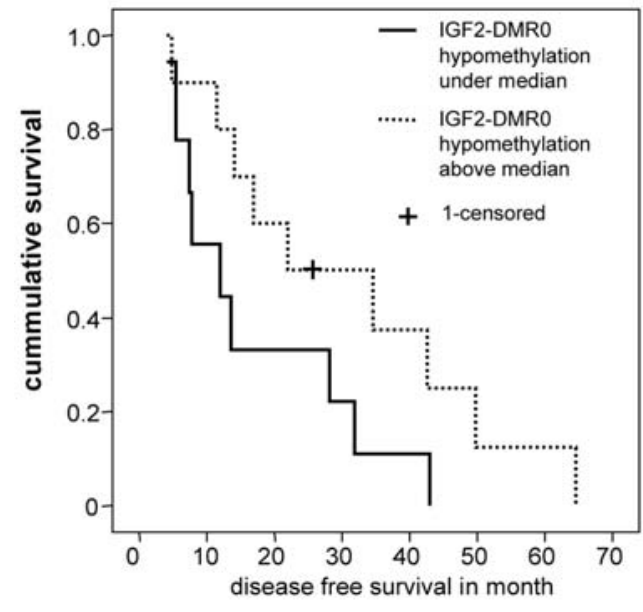

Figure 4. Kaplan-Meier survival curve. Patients were divided in those with a tumor that harboured IGF2-DMR0 hypomethylation under median and tumors with IGF2-DMR0 hypomethylation above median and the disease free survival was plotted in a Kaplan-Meier survival graph.

(Fig. 3 and Table III). CAOV3 was the only cell line with a hypermethylation of CTCF-BS6. Interestingly, we observed that patients $(n=9)$ with a hypomethylation under median of $I G F 2$-DMR had a trend for a shorter disease free survival compared to patients $(\mathrm{n}=10)$ with hypomethylation above median ( $\mathrm{p}=0.086$; Fig. 4).

LINE1 and Satellite 2 hypomethylation. To analyze the methylation status of repetitive sequences in OC, the methylation of the pericentromeric heterochromatin associated Satellite 2 DNA (Sat2) and of the retrotransposon LINE1 was investigated by QUBRA (Fig. 5A). Only five of 22 (23\%) cases showed hypomethylation of Sat2 (Fig. 5B and Table III). In contrast, hypomethylation of LINE1 was detected in all analyzed cases (Fig. 5C and Table III) and was much more pronounced in poorly differentiated OC compared to well differentiated cases (Fig. 5D).

Correlation between methylation frequency. All cases with aberrant methylation of the IGF2/H19 locus also exhibited hypomethylation of LINE1 (Table III). There was no association between methylation rates (data not shown). To analyze the effect of an inhibitor of DNA methyltransferase on the methylation status of Sat2, LINE1, IGF2-DMR0 and CTCFBS6 in normal human epithelial cells, we treated the cell line MCF10 with 5-aza-2'-deoxycytidine (5-aza-dCR) for four days and analyzed the methylation by QUBRA (Fig. 6 and Table IV). This treatment induced a hypomethylation of Sat2, LINE1 and IGF2-DMR0, however, CTCF-BS6 was not demethylated. 
Table III. Summary of methylation analysis in primary ovarial carcinoma.

\begin{tabular}{|c|c|c|c|c|c|}
\hline & Case & $\begin{array}{l}I G F 2 \text { DMR0 } \\
\text { methylation } \\
\quad( \pm \mathrm{SD})\end{array}$ & $\begin{array}{l}\text { CTCF BS6 } \\
\text { methylation } \\
\quad( \pm \mathrm{SD})\end{array}$ & $\begin{array}{l}\text { Sat2 } \\
\text { methylation } \\
( \pm \mathrm{SD})\end{array}$ & $\begin{array}{l}\text { LINE1 } \\
\text { methylation } \\
\quad( \pm \mathrm{SD})\end{array}$ \\
\hline \multirow{22}{*}{$\begin{array}{l}\text { Primary } \\
\text { ovarial } \\
\text { carcinoma (OC) }\end{array}$} & $\mathrm{OC} 2$ & $0.68(0.42)$ & 2.31 (1.97) & $1.00(0.00)$ & nd \\
\hline & OC3 & $0.41(0.08)$ & $3.63(0.58)$ & $0.23(0.10)$ & $0.22(0.04)$ \\
\hline & $\mathrm{OC} 4$ & $0.32(0.02)$ & $4.28(0.02)$ & $1.00(0.00)$ & $0.39(0.09)$ \\
\hline & OC6 & $0.40(0.16)$ & $4.29(0.00)$ & $0.82(0.06)$ & $0.44(0.06)$ \\
\hline & OC13 & $1.10(0.26)$ & $0.56(0.39)$ & $1.00(0.00)$ & nd \\
\hline & OC14 & $0.46(0.14)$ & $3.03(1.72)$ & $1.00(0.00)$ & $0.47(0.05)$ \\
\hline & OC 15 & $0.37(0.06)$ & $0.53(0.63)$ & $1.00(0.00)$ & $0.63(0.13)$ \\
\hline & OC22 & $0.23(0.00)$ & $0.03(0.03)$ & $1.00(0.00)$ & 0.83 (nd) \\
\hline & OC30 & $0.24(0.10)$ & $3.97(0.05)$ & $1.00(0.00)$ & nd \\
\hline & OC 32 & $0.07(0.02)$ & $0.04(0.01)$ & $1.00(0.00)$ & $0.52(\mathrm{nd})$ \\
\hline & OC37 & $0.19(0.08)$ & $0.42(0.57)$ & $1.00(0.00)$ & $0.76(0.03)$ \\
\hline & OC39 & $0.72(0.16)$ & $4.23(0.04)$ & $0.98(0.01)$ & $0.62(0.04)$ \\
\hline & OC41 & $0.42(0.06)$ & $2.89(0.10)$ & $1.00(0.00)$ & $0.39(0.13)$ \\
\hline & OC43 & $0.21(0.00)$ & $3.63(0.24)$ & $0.84(0.12)$ & $0.11(0.12)$ \\
\hline & OC45 & $0.21(0.11)$ & $2.51(0.11)$ & $0.80(0.05)$ & $0.52(0.12)$ \\
\hline & OC46 & $0.47(0.33)$ & $4.17(0.08)$ & $0.94(0.04)$ & $0.23(0.03)$ \\
\hline & OC49 & $0.50(0.24)$ & $1.98(1.52)$ & $0.94(0.05)$ & $0.24(0.14)$ \\
\hline & OC50 & $0.36(0.15)$ & $4.29(0.00)$ & $0.99(0.01)$ & $0.58(0.39)$ \\
\hline & OC51 & $1.24(0.09)$ & $2.91(0.39)$ & $0.99(0.01)$ & $0.10(0.09)$ \\
\hline & OC55 & $0.48(0.04)$ & $1.31(0.92)$ & $1.00(0.00)$ & $0.50(0.04)$ \\
\hline & OC56 & $0.38(0.07)$ & $0.40(0.53)$ & $0.68(0.02)$ & $0.07(0.06)$ \\
\hline & OC57 & $0.77(0.08)$ & $3.11(0.94)$ & $1.00(0.00)$ & $0.54(0.13)$ \\
\hline \multirow[t]{5}{*}{ OC cell lines } & SKOV3 & $0.08(0.01)$ & $0.43(0.14)$ & $1.00(0.00)$ & $0.48(0.18)$ \\
\hline & OAW42 & $3.33(0.02)$ & $0.03(0.04)$ & $1.00(0.00)$ & $0.53(0.16)$ \\
\hline & OVCAR3 & $0.10(0.01)$ & $0.68(0.17)$ & $0.40(0.07)$ & $0.32(0.11)$ \\
\hline & CAOV3 & $1.41(0.14)$ & $4.19(0.10)$ & $1.00(0.00)$ & $0.39(0.11)$ \\
\hline & $\mathrm{ES} 2$ & $0.72(0.02)$ & $0.29(0.03)$ & $0.69(0.05)$ & $0.48(0.02)$ \\
\hline
\end{tabular}

All methylation levels are relative to controls $(=1)$; SD, standard deviation; nd, not determined.

Table IV. Methylation of IGF2 DMR0, CTCF BS6, Sat2 and LINE1 in MCF10.

\begin{tabular}{lcccc}
\hline 5 -aza-dCR & IGF2 DMR0 $( \pm \mathrm{SD})$ & CTCF BS6 $( \pm$ SD $)$ & Sat2 $( \pm$ SD $)$ & LINE1 $( \pm$ SD $)$ \\
\hline $0 \mu \mathrm{M}$ & $1.10( \pm 0.03)$ & $0.99( \pm 0.10)$ & $1.00( \pm 0)$ & $0.84( \pm 0.00)$ \\
$5 \mu \mathrm{M}$ & $0.89( \pm 0.06)$ & $1.08( \pm 0.05)$ & $0.94( \pm 0.05)$ & $0.83( \pm 0.01)$ \\
$10 \mu \mathrm{M}$ & $0.44( \pm 0.03)$ & $0.98( \pm 0.15)$ & $0.72( \pm 0.02)$ & $0.42( \pm 0.34)$ \\
\hline
\end{tabular}

All methylation levels relative to blood samples (=1); SD, standard deviation.

\section{Discussion}

Conventional methylation specific PCR and combined bisulfite restriction analysis does not provide quantitative information on the proportion of methylated sequences among total alleles. For measurement of methylation changes that occur during loss of imprinting and during hypomethylation of repetitive
DNA elements, we established a quantitative bisulfite restriction analysis (QUBRA) of the IGF2-DMR, CTCF-BS6, Sat2 and LINE1. In contrast to other quantitative assays like Bio-COBRA (30) and pyrosequenzing, which depend on micro fluidics chips and a Bioanalyzer or a Pyrosequenzer, QUBRA is an inexpensive and reliable method and requires only standard laboratory equipment. 
$A_{\mathrm{M}} \frac{\mathrm{OC} 2}{-+} \frac{\mathrm{OC} 3}{-+} \frac{\mathrm{OC} 4}{-+} \frac{\mathrm{OC} 6}{-+} \frac{\mathrm{OC} 13}{-+} \frac{\mathrm{OC} 14}{-+} \frac{\mathrm{OC} 15}{-+} \mathrm{M} \frac{\mathrm{OC} 22}{-+} \frac{\mathrm{OC} 30}{-+} \frac{\mathrm{OC} 32}{-+} \frac{\mathrm{OC} 37}{-+} \frac{\mathrm{OC} 39}{-+}$

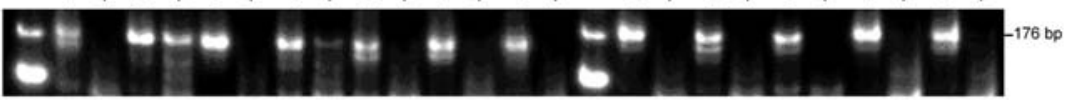

OC41 OC43 OC45 OC46 OC49 OC50 OC51 OC55 OC56 OC57 SKOV3 OAW42

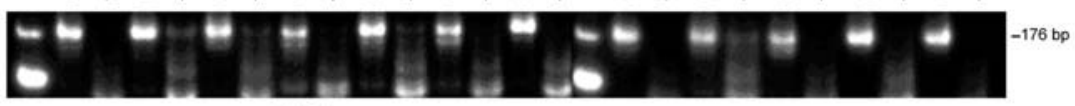

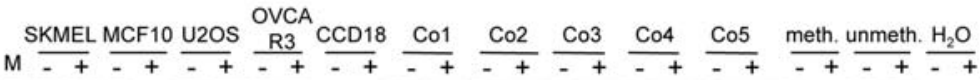

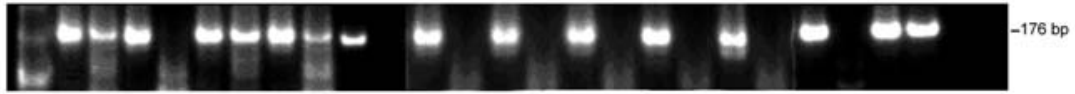

B

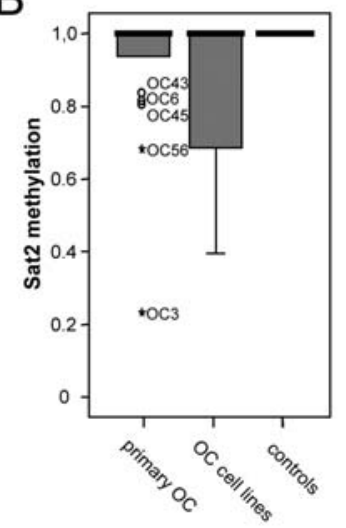

C

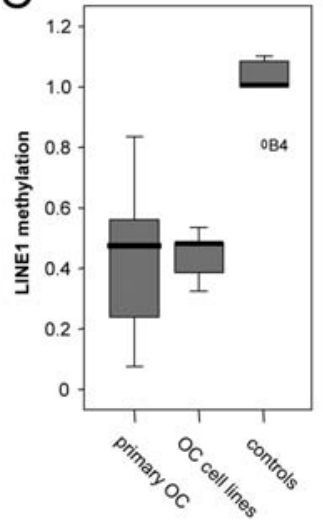

D

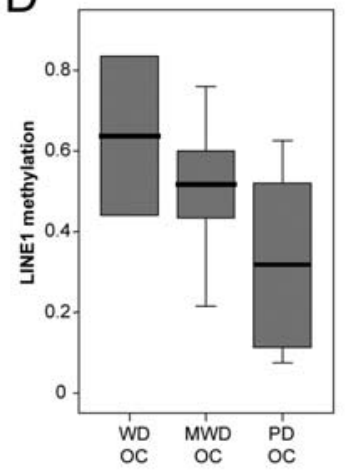

Figure 5. Methylation analysis in ovarian carcinoma. (A) Methylation of the Sat2 in primary ovarian carcinoma (OC) samples, cancer cell lines (SKOV3, OAW42, SKMEL, U2OS and OVCAR3) normal cell lines (MCF10 and CCD18) and controls (Co) was determined by QUBRA with restrictions enzyme (+) or mock digest (-). Methylated (meth.) and unmethylated (unmeth.) standards were separated together with a 100 bp ladder (M) by PAGE. (B) Whisker-boxplot of the Sat2 methylation status of primary ovarian carcinomas (OC), OC cell lines (SKOV3, OAW42, OVCAR3, CAOV3 and ES2) and controls. Methylation levels are relative to controls (=1). (C) Whisker-box-plot of LINE1 methylation in primary ovarian carcinomas (OC), OC cell lines and controls. For details see Fig. 5B. (D) Whisker-box-plot of LINE1 methylation. Methylation was associated with differentiational stage of the primary ovarian carcinoma (OC) according to well-differentiated (WD), moderately well differentiated (MWD) and poorly-differentiated carcinoma (PD).

To the best of our knowledge, this is the first study, which analyzed combined methylation status of IGF2-H19, LINE1 and Sat2 in ovarian carcinoma (OC). All primary OC exhibited LINE1 hypomethylation and in $91 \%$ of OC a significant hypomethylation of the IGF2 DMR0 was found. This result shows that hypomethylation of LINE1 is also associated with demethylation of DMR0 in exon 3 within the IGF2 gene. As already suggested by Cui et al this implies a CTCF and enhancer-independent mechanisms of IGF2 through aberrant methylation of additional regulatory sequences within the IGF2 gene (9). The DMR0 has been shown to contain promoter activity (31). Thus, hypomethylation of IGF2-DMR, leads to $I G F 2$ overexpression in colon cancer and is associated with up to 5-fold increased risk of colorectal neoplasia $(7,8)$. In ovarian cancers, maternal allele-specific hypomethylation in IGF2-DMR were more likely detectable in tumor cells when compared to normal lymphocyte DNA (5) and this is consistent with our results. IGF2-DMR0 hypomethylation was also observed in lymphocytes of the normal population $(8,19)$. We observed hypomethylation of CTCF-BS6 in blood controls (Fig. 3C) and therefore four times higher methylation was observed in some of the OCs.

We observed that patients with a pronounced hypomethylation of IGF2-DMR0 had a trend for a shorter relapse-
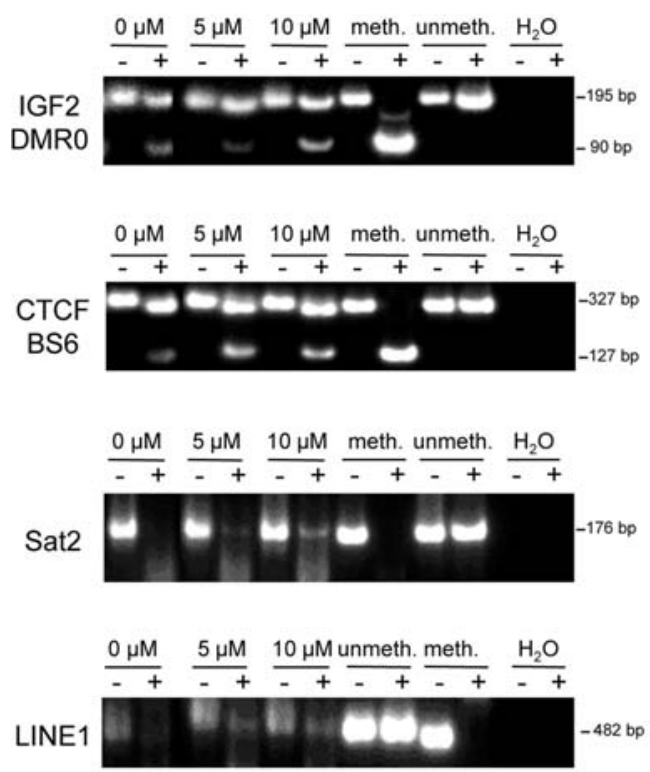

Figure 6. Methylation analysis of MCF10 cell treated with 5-aza-dCR. Human mammary epithelial cells were treated for 4 days with indicated concentrations of 5-aza-dCR and methylation status of IGF2-DMR0, CTCF-BS6, Sat2 and LINE1 was analyzed by QUBRA with restrictions enzyme (+) or mock digested (-). Methylated (meth.) and unmethylated (unmeth.) standards and a negative control $\left(\mathrm{H}_{2} \mathrm{O}\right)$ were utilized as controls. 
free survival compared to the patients with a weaker hypomethylation $(\mathrm{p}=0.086)$. Thus, our finding indicates that methylation status of IGF2 may have prognostic relevance in OC. However, in a study were only a qualitative methylation status of the IGF2-DMR was revealed, no correlation to survival was found (5). Thus the degree of IGF2-DMR hypomethylation could be critical for survival analysis, but this has to be validated in a larger cohort of patients.

The hypermethylation of CTCF-BS6 was observed in $77 \%$ of OC compared to the controls $(\mathrm{p}<0.05)$. This finding is in concordance with the enhancer competition model of IGF2 and H19 promoters for a shared enhancer. The accessibility of the maternal IGF2 allele to this enhancer is blocked by CTCF binding to unmethylated CTCF binding sites on $H 19$ (i.a. CTCF-BS6) (32). Accordingly, relaxation of IGF2 imprinting could be the result of hypermethylation of CTCFBS6. It is well known that removal of IGF2 imprinting in OC may be initiated by methylation and blocking of CTCF binding sites on $H 19$ (32). In serous epithelial ovarian cancer, a positive correlation between elevated $I G F 2$ expression and hypermethylation of CTCF-binding site was observed previously $(5,33)$. In our study, we detected frequent combined hypomethylation of IGF2-DMR and hypermethylation of CTCF-BS6 indicating a simultaneous or related loss of imprinting at the IGF2-H19 DMR and ICR.

The methylation of the pericentromeric heterochromatin associated Satellite 2 DNA and of the retrotransposon LINE1 was investigated by QUBRA. LINE1 was hypomethylated in all OC. High prevalence of LINE1 hypomethylation throughout all tumor stages and grades allows the conclusion that hypomethylation of LINE1 is an early event during ovarian carcinogenesis. Previous studies reported that LINE1 hypomethylation was associated with FIGO stage (the International Federation of Gynecology and Obstetrics) and tumor grade in OC (34). We observed more pronounced LINE1 hypomethylation in poorly differentiated OC compared to well differentiated cases. However, only $23 \%$ of OC exhibited hypomethylation in Sat 2 region. The low frequence of hypomethylation in Sat2 DNA might be a consequence of a hypoacetylated heterochromatin and suggests a low degree of pericentromeric rearrangements in the investigated OC (35). Treatment of cells with 5-aza-dCR induced hypomethylation of Sat2, LINE1 and IGF2-DMR0, however CTCF-BS6 was not demethylated. This result may indicate a different mechanism to maintain the methylation state at the CTCF binding site and that germ line specific DMR could be resistant to demethylation.

We have generated a quantitative method to investigate aberrant methylation changes at the IGF2/H19 locus, LINE1 and Sat2 in ovarian carcinoma (OC). Combined hypomethylation of the IGF2 DMR0 and LINE1 were frequently detected. Interestingly, hypomethylation of Sat2 was infrequently found and hypermethylation of a CTCF binding site occurred in these OC. These results indicate a specific hypomethylation of distinct elements (LINE1 and DMR0) and hypermethylation of others (CTCF-BS) rather than global hypomethylation or hypermethylation of genomic DNA. Thus, mechanisms that produce these divergent methylation patterns in the same tumor should be revealed in more detail.

\section{Acknowledgements}

Reinhard Dammann received grant support from BMBF (FKZ 01ZZ0104), Deutsche Krebshilfe (109179) and DFG (DA552).

\section{References}

1. Bird A: DNA methylation patterns and epigenetic memory. Genes Dev 16: 6-21, 2002.

2. Ravenel JD, Broman KW, Perlman EJ, et al: Loss of imprinting of insulin-like growth factor-II (IGF2) gene in distinguishing specific biologic subtypes of Wilms tumor. J Natl Cancer Inst 93: 1698-1703, 2001.

3. Sullivan MJ, Taniguchi T, Jhee A, Kerr N and Reeve AE: Relaxation of IGF2 imprinting in Wilms tumours associated with specific changes in IGF2 methylation. Oncogene 18: 7527-7534, 1999.

4. Kim HT, Choi BH, Niikawa N, Lee TS and Chang SI: Frequent loss of imprinting of the H19 and IGF-II genes in ovarian tumors. Am J Med Genet 80: 391-395, 1998.

5. Murphy SK, Huang Z, Wen Y, et al: Frequent IGF2/H19 domain epigenetic alterations and elevated IGF2 expression in epithelial ovarian cancer. Mol Cancer Res 4: 283-292, 2006.

6. Sayer RA, Lancaster JM, Pittman J, et al: High insulin-like growth factor-2 (IGF-2) gene expression is an independent predictor of poor survival for patients with advanced stage serous epithelial ovarian cancer. Gynecol Oncol 96: 355-361, 2005.

7. Cruz-Correa M, Cui H, Giardiello FM, et al: Loss of imprinting of insulin growth factor II gene: a potential heritable biomarker for colon neoplasia predisposition. Gastroenterology 126: 964-970, 2004.

8. Cui H, Cruz-Correa M, Giardiello FM, et al: Loss of IGF2 imprinting: a potential marker of colorectal cancer risk. Science 299: 1753-1755, 2003.

9. Cui H, Onyango P, Brandenburg S, Wu Y, Hsieh CL and Feinberg AP: Loss of imprinting in colorectal cancer linked to hypomethylation of H19 and IGF2. Cancer Res 62: 6442-6446, 2002.

10. Nakagawa H, Chadwick RB, Peltomaki P, Plass C, Nakamura Y and de La Chapelle A: Loss of imprinting of the insulin-like growth factor II gene occurs by biallelic methylation in a core region of H19-associated CTCF-binding sites in colorectal cancer. Proc Natl Acad Sci USA 98: 591-596, 2001.

11. Takano Y, Shiota G and Kawasaki H: Analysis of genomic imprinting of insulin-like growth factor 2 in colorectal cancer. Oncology 59: 210-216, 2000.

12. Jarrard DF, Bussemakers MJ, Bova GS and Isaacs WB: Regional loss of imprinting of the insulin-like growth factor II gene occurs in human prostate tissues. Clin Cancer Res 1: 1471-1478, 1995.

13. Ulaner GA, Vu TH, Li T, et al: Loss of imprinting of IGF2 and H19 in osteosarcoma is accompanied by reciprocal methylation changes of a CTCF-binding site. Hum Mol Genet 12: 535-549, 2003.

14. Kohda M, Hoshiya H, Katoh M, et al: Frequent loss of imprinting of IGF2 and MEST in lung adenocarcinoma. Mol Carcinog 31: 184-191, 2001.

15. El-Naggar AK, Lai S, Tucker SA, et al: Frequent loss of imprinting at the IGF2 and H19 genes in head and neck squamous carcinoma. Oncogene 18: 7063-7069, 1999.

16. Rainho CA, Kowalski LP and Rogatto SR: Loss of imprinting and loss of heterozygosity on $11 \mathrm{p} 15.5$ in head and neck squamous cell carcinomas. Head Neck 23: 851-859, 2001.

17. Ohlsson R, Nystrom A, Pfeifer-Ohlsson S, et al: IGF2 is parentally imprinted during human embryogenesis and in the Beckwith-Wiedemann syndrome. Nat Genet 4: 94-97, 1993.

18. Vu TH, Li T, Nguyen D, et al: Symmetric and asymmetric DNA methylation in the human IGF2-H19 imprinted region. Genomics 64: 132-143, 2000.

19. Ito Y, Koessler T, Ibrahim AE, et al: Somatically acquired hypomethylation of IGF2 in breast and colorectal cancer. Hum Mol Genet 17: 2633-2643, 2008.

20. Kurukuti S, Tiwari VK, Tavoosidana G, et al: CTCF binding at the H19 imprinting control region mediates maternally inherited higher-order chromatin conformation to restrict enhancer access to Igf2. Proc Natl Acad Sci USA 103: 10684-10689, 2006. 
21. Chalitchagorn K, Shuangshoti S, Hourpai N, et al: Distinctive pattern of LINE-1 methylation level in normal tissues and the association with carcinogenesis. Oncogene 23: 8841-8846, 2004.

22. Ostertag EM and Kazazian HH Jr: Biology of mammalian L1 retrotransposons. Annu Rev Genet 35: 501-538, 2001.

23. Qu G, Dubeau L, Narayan A, Yu MC and Ehrlich M: Satellite DNA hypomethylation vs. overall genomic hypomethylation in ovarian epithelial tumors of different malignant potential. Mutat Res 423: 91-101, 1999.

24. Saito Y, Kanai Y, Sakamoto M, Saito H, Ishii H and Hirohashi S: Expression of mRNA for DNA methyltransferases and methylCpG-binding proteins and DNA methylation status on CpG islands and pericentromeric satellite regions during human hepatocarcinogenesis. Hepatology 33: 561-568, 2001.

25. Ehrlich M, Hopkins NE, Jiang G, et al: Satellite DNA hypomethylation in karyotyped Wilms tumors. Cancer Genet Cytogenet 141: 97-105, 2003.

26. Kazazian HH Jr: Mobile elements: drivers of genome evolution. Science 303: 1626-1632, 2004

27. Menendez L, Benigno BB and McDonald JF: L1 and HERV-W retrotransposons are hypomethylated in human ovarian carcinomas. Mol Cancer 3: 12, 2004.

28. Suter CM, Martin DI and Ward RL: Hypomethylation of L1 retrotransposons in colorectal cancer and adjacent normal tissue. Int J Colorectal Dis 19: 95-101, 2004.

29. Widschwendter M, Jiang G, Woods C, et al: DNA hypomethylation and ovarian cancer biology. Cancer Res 64: 4472-4480, 2004.
30. Brena RM, Auer H, Kornacker K and Plass C: Quantification of DNA methylation in electrofluidics chips (Bio-COBRA). Nat Protoc 1: 52-58, 2006.

31. Monk D, Sanches R, Arnaud P, et al: Imprinting of IGF2 P0 transcript and novel alternatively spliced INS-IGF2 isoforms show differences between mouse and human. Hum Mol Genet 15: 1259-1269, 2006.

32. Pant V, Kurukuti S, Pugacheva E, et al: Mutation of a single CTCF target site within the H19 imprinting control region leads to loss of Igf2 imprinting and complex patterns of de novo methylation upon maternal inheritance. Mol Cell Biol 24: 34973504, 2004.

33. Chen CL, Ip SM, Cheng D, Wong LC and Ngan HY: Loss of imprinting of the IGF-II and H19 genes in epithelial ovarian cancer. Clin Cancer Res 6: 474-479, 2000.

34. Pattamadilok J, Huapai N, Rattanatanyong P, et al: LINE-1 hypomethylation level as a potential prognostic factor for epithelial ovarian cancer. Int J Gynecol Cancer 18: 711-717, 2008.

35. Johnson CA, O'Neill LP, Mitchell A and Turner BM: Distinctive patterns of histone $\mathrm{H} 4$ acetylation are associated with defined sequence elements within both heterochromatic and euchromatic regions of the human genome. Nucleic Acids Res 26: 994-1001, 1998. 